\title{
Dynamics of nonlinear waves in the tubes filled with aerosol
}

\author{
Damir Gubaidullin ${ }^{1, *}$, Rinat Zaripov ${ }^{1}$, and Liudmila Tkachenko ${ }^{1}$ \\ ${ }^{1}$ Institute of Mechanics and Engineering, Kazan Science Center, Russian Academy of Sciences, 420111 Kazan, Russia
}

\begin{abstract}
The results of experimental investigations of nonlinear oscillations of finely dispersed aerosol in the tube with various geometry on the end in the shock-wave, the shock-free wave modes and in the mode of transition to shock waves near the resonance frequency are presented. The time dependences of the numerical concentration of the oscillating aerosol droplets are presented. The effect of the frequency and amplitude of the piston displacement and the influence of the diaphragm internal diameter on the time coagulation and sedimentation of aerosol were studied. An increase in the amplitude of the piston displacement in all modes results in acceleration of the process of coagulation and sedimentation of aerosol. The dependence of time of coagulation and sedimentation of aerosol on the excitation frequency was found to be of a nonmonotonic character with the minimum value upon the resonance frequency.
\end{abstract}

\section{INTRODUCTION}

Various inhomogeneous media with droplets or solid particles are the main working bodies in many technological processes. The study of the wave processes in such media is one of important problems in the dynamics and thermophysics of multiphase systems [1]. Technologies created on the basis of the wave principle can be used for cleaning of gases, including hightemperature (flue, smoke) and radioactive from solid fine-dispersed inclusions; for sedimentation of aerosols, mists, including toxic, chemically reactive in limited volumes; for trapping of water vapor and other liquids in thermal plants, in particular, in cooling towers. The study of coagulation and sedimentation of aerosols under influence of wave acoustic fields is of great importance for the development of such technologies [2-4]. Some results on the coagulation and sedimentation of aerosols at resonance oscillations in tubes with different conditions on the ends are considered in experimental works [5-15].

In this paper, detailed results of experimental studies of the dynamics of nonlinear waves in closed and open tubes filled with aerosol are presented in the shock-wave and the shock-free wave modes and in the mode of transition to shock waves near the resonance frequencies.

\section{NONLINEAR OSCILLATIONS OF AEROSOL IN TUBES IN THE SHOCK- WAVE MODE}

\subsection{Experimental setup}

The experimental setup consisted of tube-resonator, generator of aerosol oscillations, oscillation frequency measuring unit containing frequency transducer and frequency meter, photo-transducer registering lightpenetrability of medium, aerosol generator, laser spectrometer, combined multimeter and digital chamber. Longitudinal oscillations of aerosol column inside the tube with different length of $L_{0}=2.7,3.7,4.7,6.7 \mathrm{~m}$ and inner diameter $2 R_{0}=0.048 \mathrm{~m}$ were created by the flat piston. The tube is made of stainless sections and was joined to the cylinder head with inner diameter $2 R=0.077 \mathrm{~m}$ through a cone reducer of height $h=0.22$ $\mathrm{m}$. The cone reducer serves to obtain greater forced aerosol oscillations in comparison with the tube of uniform cross-section. One section in length of $1.2 \mathrm{~m}$ has been made of a quartz glass and established in the middle of the tube in experiment with the closed tube and on distance of $1.87 \mathrm{~m}$ from cone reducer in experiment with the open tube. The photo-transducer attached to the quartz section of the tube permitted us to register the aerosol transparency at different particle concentration of droplets in a medium. The excitation frequency $v$ varied from 0 to $18 \mathrm{~Hz}$ and was controlled by the frequency meter with accuracy of $0.1 \mathrm{~Hz}$. Aerosol is turned out from the liquid Di-ethyl-hexyl-sebacate $\mathrm{C}_{26} \mathrm{H}_{50} \mathrm{O}_{4}$ (DEHS) by means of ATM 225 (TOPAS) aerosol generator. Average geometrical diameter droplets of aerosol in undisturbed state were measured by LAP 320 laser spectrometer (TOPAS) and equalled $0.863 \mu \mathrm{m}$ (Figure 1).

\subsection{Experimental results}

As a result of experimental researches dependences of numerical concentration of droplets of aerosol on time have been received. They are approximated by dependence

$$
N=A_{0}+A_{1} \exp \left(-K_{1} t\right)+A_{2} \exp \left(-K_{2} t\right)
$$

\footnotetext{
${ }^{*}$ Corresponding author: damirgubaidullin@gmail.com
} 


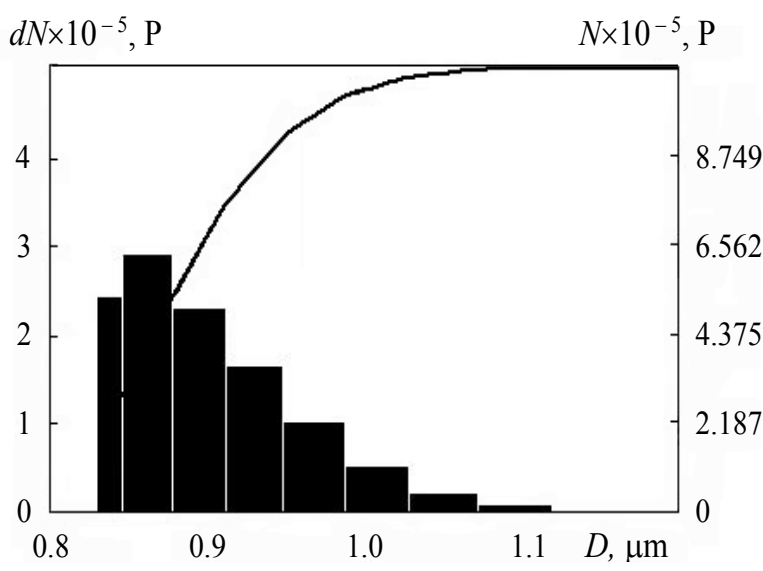

Fig. 1. Distribution of the unperturbed weighed phase of a dispersion medium on the geometrical diameter.

where coefficients are from experiment. First two terms well describe experimental data at oscillations of aerosol far from a subharmonic resonance. The third term takes into account nonlinear effects in a resonance (shock waves, secondary flows, pulsing jet of aerosol from the open end). Concentration of droplets of aerosol for all experiments monotonously decreases in due course. The above frequency of excitation, the occurs this process faster. In the closed tube, the process of decrease of droplets concentration of aerosol in due course includes coagulation of droplets and their sedimentation on walls of the tube. In the open tube, the process of decrease of concentration of the droplets of aerosol in due course includes basically coagulation of droplets, their sedimentation on walls of the tube, and also nonstationary emission of aerosol from the open end of the tube as a pulsing jet. The time of coagulation and sedimentation of aerosol in the closed tube in the shockwave mode on subharmonic resonances is up to 5 times less, than at the natural sedimentation. In the open tube, the natural sedimentation lasts up to 30 times more slowly than time of coagulation and sedimentation of aerosol in the shock-wave mode.

Dependences of time of coagulation and sedimentation of aerosol on the excitation frequency for different values of length of the closed tube are given in Figure 2. Every point in the curves corresponds to the certain experiment. It is seen from the Figure 2 that for tubes of $L_{0}=2.7,3.7,4.7 \mathrm{~m}$ length with increasing frequency in pre-resonant regime, time of coagulation and sedimentation of aerosol decreases. It takes place due to the fact that as the frequency approaches the resonance aerosol oscillation amplitude increases. For the tube of $6.7 \mathrm{~m}$ length, the minimum of time of coagulation and sedimentation of aerosol occurs at the $12 \mathrm{~Hz}$ frequency, afterwards it starts to increase. It is a matter of fact that at this frequency, subharmonic resonance occurs in the tube with a frequency two times lower as the fundamental one of a medium $v_{1 / 2}=c_{0} / 4 L$ $\left(c_{0}\right.$ is the equilibrium velocity of sound in aerosol [16], and $L=L_{0}+l+\left(m^{2}+m+1\right) h / 3$ is the reduced tube length, where $\left.l=m^{2} l_{0}, \quad m=R / R_{0}\right)$, the oscillation amplitude reaching maximum at this frequency. For tubes of $L_{0}=2.7,3.7,4.7 \mathrm{~m}$ length, the resonant frequencies lie higher than $18 \mathrm{~Hz}$, therefore they were not reached in the present experiment.

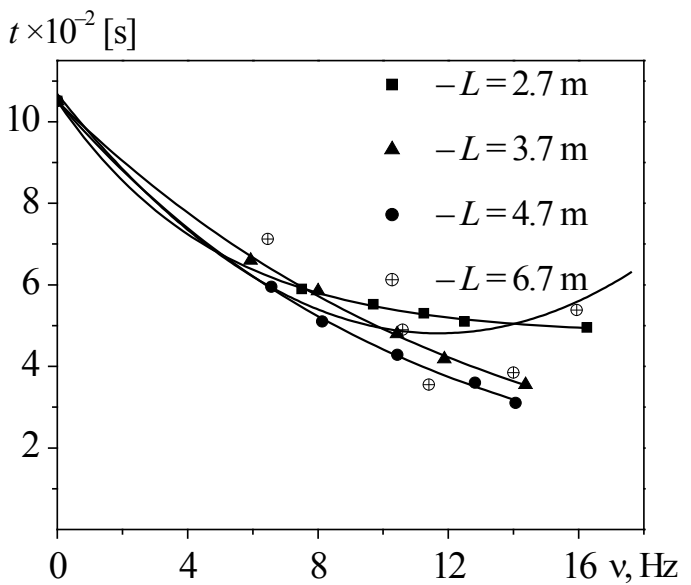

Fig. 2. Dependences of time of coagulation and sedimentation of the aerosol on frequency in the closed tube. The points indicate experimental data, and the solid lines indicate the polynomial approximation.

On Figure 3 dependencies of time of coagulation and sedimentation of aerosol on frequency of excitation for various lengths of the open tube are given. Note that the curve for the tube of $L_{0}=4.7 \mathrm{~m}$ length has a minimum and a maximum. The minimum corresponds to frequency $9 \mathrm{~Hz}$ of a subharmonic resonance $v_{1 / 2}=$ $=c_{0} / 8 L\left(L=L_{0}+l+\left(m^{2}+m+1\right) h / 3+\sigma R_{0}\right.$, where $\sigma R_{0}$ Rayleigh correction with $\sigma=0.6133)$. Behind the resonance time of coagulation and sedimentation of aerosol increases up to the maximum and then quickly decreases. Thus, time of coagulation and sedimentation of aerosol in the open tube decreases in two and more times in comparison in due course coagulations and sedimentation in the closed tube.

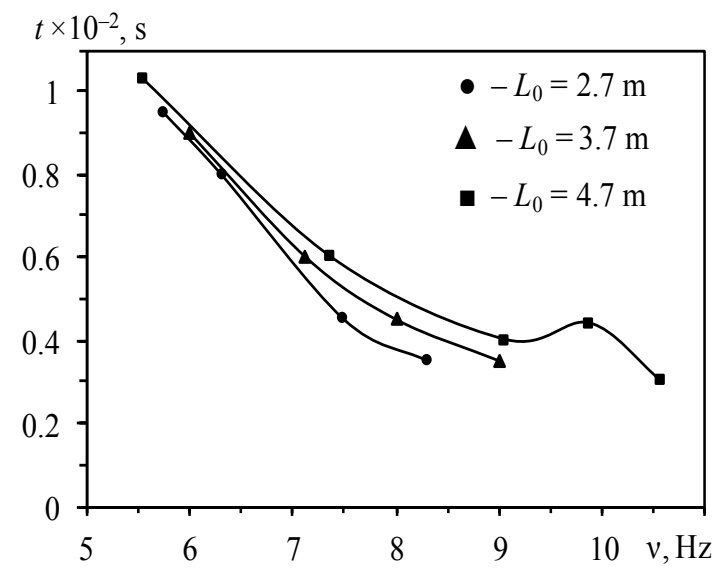

Fig. 3. Dependences of time of coagulation and sedimentation of aerosol on frequency in the open tube. The points indicate the experimental data, and the solid lines indicate the polynomial approximation.

Also, influence of the diaphragms at the passive end of the tube on the process of coagulation and sedimentation of aerosol is studied. In Figure 4 the dependence of time of coagulation and sedimentation of aerosol on the excitation frequency for the closed and the 
open tubes without diaphragm, as well as the open tube with diaphragms of different internal diameters is given. Figure 4 shows that decrease in the internal diameter of the diaphragm $2 \mathrm{R}_{\text {in.f }}$ results in the increase of time of coagulation and sedimentation of aerosol, which reaches the maximum value in the case of the closed tube. This is due, firstly, to significant influence of the unsteady discharge of aerosol from the open end of the tube, and secondly, with the approach of the investigated excitation frequencies of aerosol in the open tube to its resonance frequency $[17,18]$. Note that time of coagulation and sedimentation of aerosol in the open tube with the diaphragm is longer than in the open tube without a diaphragm. This is due to the fact that the open tube with the diaphragm which is in connection with the tube forms a right angle, the consumption on the formation of vortexes near the outlet section is greater than in the tube with a rounded open end [19].

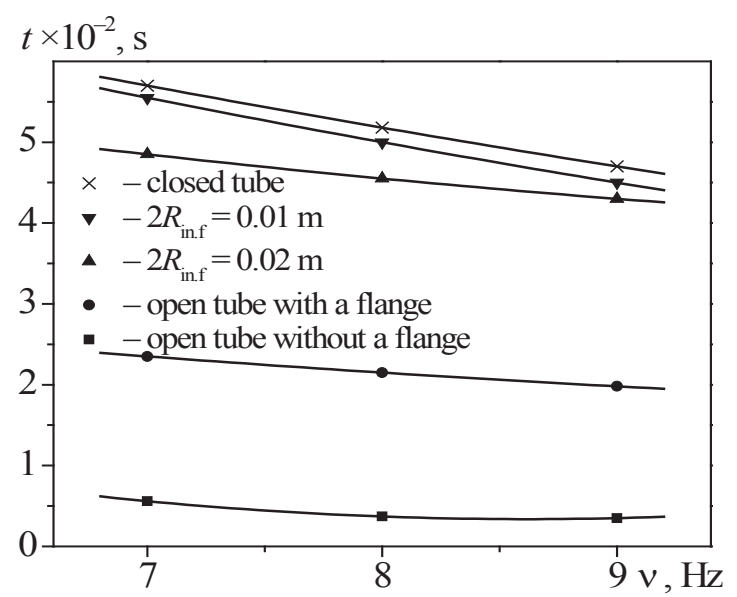

Fig.4. Dependence of time of coagulation and sedimentation of aerosol on frequency in to-resonance regime for the tube of $L_{0}=$ $2.7 \mathrm{~m}$ length with diaphragms of different internal diameters. The points indicate the experimental data, and the solid lines indicate the polynomial approximation.

\section{DYNAMICS OF AEROSOL AT OSCILLATIONS IN THE SHOCK-FREE WAVE MODE AND IN THE MODE OF TRANSITION TO SHOCK WAVES}

\subsection{Experimental setup}

The investigation was carried out on an experimental setup containing the tube, the system of oscillations excitation, and the system of detection of process parameters. The oscillations of aerosol in closed and open tubes were excited with the flat piston. Studies in the shock-free wave mode were carried out in the tube $L=1.06 \mathrm{~m}$ long and $R_{0}=0.0365 \mathrm{~m}$ in radius using TIRAvib 51075 vibrostend, and in the mode of transition to shock waves in the tube $L=0.938 \mathrm{~m}$ long and $R_{0}=0.1$ $\mathrm{m}$ in radius using TIRAvib $\mathrm{S}$ 5220/LS vibrostend. The measurements and control of sinusoidal oscillations were performed using software module installed on computer, by means of the integrated electronic piezoelectric accelerometer and aerosol, we used 8530C-15 (Bruel and Kjaer) pressure gauge with ENDEVCO-136 (Bruel and Kjaer) three-channel bridge voltage amplifier and DSO 3062A (Agilent Technologies) digital oscillograph. For determining of aerosol transparency, we used the complex consisting of the light source and ATT-1505 (Aktakom) digital luxmeter. As before, DEHS was used as the operating liquid.

\subsection{Experimental results}

The time dependences of pressure oscillations and numerical concentration of aerosol droplets were obtained for various values of amplitude of the piston displacement near the first eigenfrequency. In the shockfree wave mode, this frequency is equal to $160.7 \mathrm{~Hz}$ in the closed tube and $78.6 \mathrm{~Hz}$ in the open tube and in the mode of transition to shock waves, $182 \mathrm{~Hz}$ and $90.3 \mathrm{~Hz}$, respectively. In all these cases, numerical concentration of aerosol decreases monotonically with time, as in the case of the shock-wave mode. This decrease is faster with increase of the amplitude. For the natural sedimentation, a smoother decrease in time for aerosol concentration is observed. The process time exceeds 2-4 and 6-12 times that in the presence of oscillations in the shock-free wave mode and exceeds 2.5-5 and 3-18 times that in the presence of oscillations in the mode of transition to shock waves, in the closed and the open tubes, respectively. As in before, we accepted that time of change in the numerical concentration in the tubes is the time of coagulation and sedimentation of aerosol.

In Figure 5, we plot the dependences of the specified times on the relative frequency of excitation (a) and also present the diagrams of oscillations of aerosol pressure for the closed and the open tubes at the first eigenfrequency (b) in the shock-free wave mode for amplitude of the piston displacement $l=0.35 \mathrm{~mm}$. Figure 6 shows the similar dependence for the mode of transition to shock waves for amplitude of the piston displacement $l=0.3 \mathrm{~mm}$.

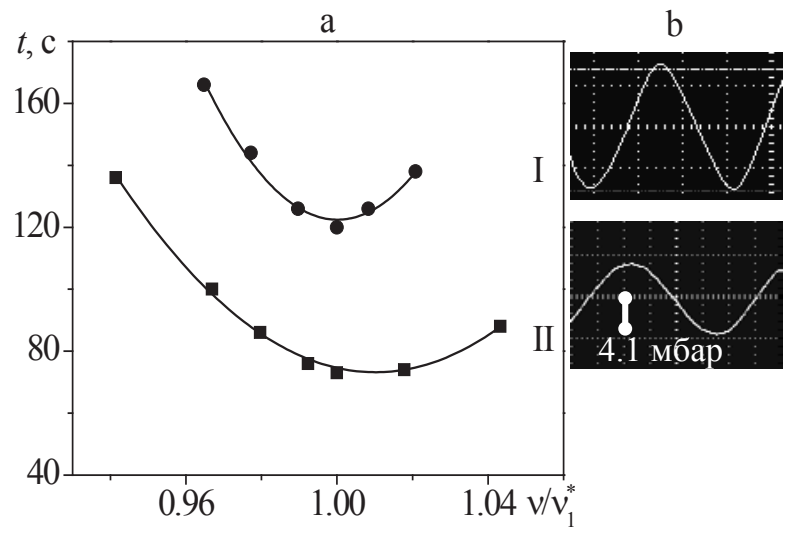

Fig.5. Dependences of time of coagulation and sedimentation of aerosol on the frequency (a) and aerosol pressure oscillations in time near the piston (b) for (I) closed and (II) open tubes in the shock-free wave mode. The lines show the polynomial approximation. 
a

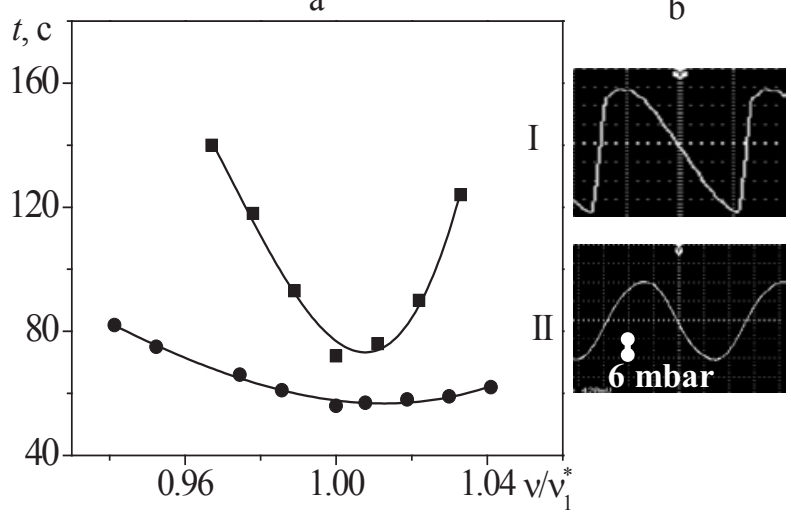

Fig.6. Dependences of time of coagulation and sedimentation of aerosol on the frequency (a) and aerosol pressure oscillations in time near the piston (b) for (I) closed and (II) open tubes in the mode of transition to shock. The lines show the polynomial approximation.

The dependences of time of coagulation and sedimentation of aerosol on the frequency have a nonmonotonic character with the minimum at the first eigenfrequency, when the amplitude of aerosol pressure oscillations is the largest. In the closed tube at the shockfree wave mode deformation of the pressure-wave shape (compression time is less the rarefaction time) is observed. In the open tube, both in the shock-free wave and in the mode of transition to shock waves the pressure-wave shape is close to a sine wave even in the resonance. In these cases, the change in time of coagulation and sedimentation of aerosol upon approaching the first eigenfrequency is insignificant. At the same time, a sharp decrease in time of coagulation and sedimentation of aerosol in the closed tube in the mode of transition to shock waves, when the pressurewave shape is close to discontinuous, occurs. In both cases, this time in the open tube is approximately 1.5 times less than in the closed tube. Such an insignificant difference in times of the processes is associated with the fact that, although a part of aerosol is thrown out into the medium in the open tube, as was already noted above, distortion of the aerosol-pressure-wave shape takes place in the closed tube. It is possible to note that, in the mode of transition to shock waves, the time of coagulation and sedimentation of aerosol in the closed and the open tubes is 1.5 times less than the corresponding time in the shock-free wave mode of oscillations for a practically identical amplitude of the piston displacement.

\section{CONCLUSIONS}

Time of coagulation and sedimentation of aerosol decreases with increase in the intensity of oscillations caused by increase in the amplitude of the piston displacement or decrease in the length of the tube and with growth in the internal diameter of the diaphragm at the passive end of the tube. The dependence of time of coagulation and sedimentation of aerosol on the excitation frequency was found to be of a nonmonotonic character with the minimum value upon the resonance frequency. At nonlinear resonance oscillations of aerosol in the mode of transition to shock waves, more efficient coagulation and sedimentation of aerosol are observed than in the shock-free wave mode at the same small amplitudes of the piston displacement. In the shockwave mode (at the large amplitudes of the piston displacement), the process is accelerated even more.

The study was performed by a grant from the Russian Science Foundation (project No. 15-11-20022).

\section{References}

1. R. I. Nigmatulin, Dynamics of Multiphase Media (Hemisphere, New York, 1990)

2. E. P. Mednikov, Acoustic Coagulation and Precipitation of Aerosols (Consultant Bureau, New York, 1965)

3. N. A. Fuks, Aerosol Mechanics (USSR Acad. Sci., Moscow, 1955)

4. S. Temkin, Suspension Acoustics: an Introduction to the Physics of Suspensions (Cambridge University Press, New York, 2005)

5. A. I. Gulyaev, V. M. Kuznetsov, Sov. Phys. Acoust. 8, 4, 236-238 (1963)

6. S. Temkin, Phys. Fluids 13, 1639-1641 (1970)

7. K. Shuster, M. Fichman, A. Goldshtein, C. Gutfinger, Phys. Fluids 14, 5, 1802-1805 (2002)

8. D. A. Gubaidullin, R. G. Zaripov, R. G. Galiullin, E. R. Galiullina, L. A. Tkachenko, High Temperature 42, 5, 794-802 (2004)

9. D. A. Gubaidullin, R. G. Zaripov, L. A. Tkachenko, High Temperature 50, 4, 564-566 (2012)

10. D. A. Gubaidullin, R. G. Zaripov, L. A. Tkachenko, High Temperature 51, 6, 955-957 (2013)

11. D. A. Gubaidullin, R. G. Zaripov, L. A. Tkachenko, Doklady Physics 58, 9, 392-395 (2013)

12. D. A. Gubaidullin, R. G. Zaripov, L. A. Tkachenko, J. of Engin. Physics and Thermophysics 85, 2, 259264 (2012)

13. D. A. Gubaidullin, R. G. Zaripov, L. A. Tkachenko, High Temperature 52, 6, 895-899 (2014)

14. D. A. Gubaidullin, R. G. Zaripov, L. A. Tkachenko, L. R. Shaidullin, High Temperature 55, 3, 463-465 (2017)

15. D. A. Gubaidullin, R. G. Zaripov, L. A. Tkachenko, Doklady Physics 62, 7, 363-365 (2017)

16. M. A. Ilgamov, R. G. Zaripov, R. G. Galiullin, V. B. Repin, Appl. Mech. Rev. 49, 3, 137-154 (1996)

17. J. Jimenez, Fluid Mech. 59. 23-46 (1973)

18. B. B. Sturtevant, J. Fluid Mech. 63, 97-120 (1974)

19. B. B. Sturtevant and J. J. Keller, Z. Angew. Math. Physics 29, 3, 473-485 (1978) 